\title{
Samambaias e licófitas do Parque Ecológico do Gunma, município de Santa Bárbara do Pará, estado do Pará, Brasil
}

\author{
Ferns and lycophytes from Gunma Ecological Park,
} Santa Bárbara do Pará municipality, Pará state, Brazil

Jeferson Miranda Costa $^{1} \&$ Marcio Roberto Pietrobom ${ }^{2}$

\begin{abstract}
Resumo
O Parque Ecológico do Gunma - PEG (Santa Bárbara do Pará, estado do Pará, Brasil) representa um dos poucos remanescentes florestais da região metropolitana de Belém. O PEG mantém uma rica flora fanerogâmica composta por espécies consideradas raras, mas nada se conhece a respeito de sua flora de plantas vasculares sem sementes. Assim, este trabalho teve como objetivo inventariar as espécies de samambaias e licófitas do Parque Ecológico do Gunma. No PEG foram registradas 56 espécies, distribuídas em 28 gêneros e 15 famílias. Das espécies registradas, Lellingeria suspensa (L.) A.R. Sm. \& R.C. Moran foi citada pela primeira vez para o estado do Pará, e Polyphlebium diaphanum (Kunth) Ebihara \& Dubuisson constituiu um novo registro para a Região Norte (Amazônia brasileira). As famílias mais representativas foram Hymenophyllaceae (15 spp.), Dryopteridaceae (8 spp.), Polypodiaceae (7 spp.) e Pteridaceae (6 spp.), e os gêneros foram Trichomanes (7 spp.) e Elaphoglossum (6 spp.). A maioria das espécies apresentou hábito herbáceo (52 spp.), habitat epifítico (30 spp.) e terrestre (23 spp.), ocorreu exclusivamente nos sub-bosques (35 spp.) e apresentou distribuição americana (34 spp.). Estes resultados demonstraram a importância do Parque Ecológico do Gunma para a preservação da flora local e regional de plantas vasculares sem sementes.

Palavras-chave: Amazônia brasileira, florística, plantas vasculares sem sementes.
\end{abstract}

\begin{abstract}
The Gunma Ecological Park - PEG (Santa Bárbara do Pará, Pará state, Brazil) is one of the few preserved forest remnants in the metropolitan region of Belém. The PEG has a rich phanerogamic flora with rare species, but nothing is known about its seedless vascular-plant flora. Our aim is to present a floristic survey of ferns and lycophytes from Gunma Ecological Park. The 56 recorded species belong to 28 genera and 15 families. This is the first record for Lellingeria suspensa (L.) A.R. Sm. \& R.C. Moran in Pará state and also for Polyphlebium diaphanum (Kunth) Ebihara \& Dubuisson in the Brazilian Amazon. The richest families were Hymenophyllaceae (15 spp.), Dryopteridaceae (8 spp.), Polypodiaceae (7 spp.) and Pteridaceae (6 spp.). The genera with highest number of species were Trichomanes (7 spp.) and Elaphoglossum (6 spp.). The majority of the species were herbaceous (52 spp.), with epiphytic (30 spp.) and terrestrial (23 spp.) habitat, occurred exclusively in the understory (35 spp.) and presented American distribution (34 spp.). This result demonstrates the importance of Gunma Ecological Park for local/regional seedless vascular-plant flora preservation.
\end{abstract}

Key words: Brazilian Amazon, floristic, seedless vascular plants.

\section{Introdução}

Estima-se que existam cerca de 13.600 espécies de samambaias e licófitas no mundo, das quais 3.500 ocorrem na América do Sul (Moran 2008). No Brasil, devem ocorrer aproximadamente
1.150 espécies (Windisch 1996a), com cerca de 550 delas podendo ser registradas na Amazônia brasileira (Prado \& Moran 2009).

As samambaias e licófitas distribuem-se dos trópicos até próximo aos círculos polares, ocorrendo

${ }^{1}$ Instituto Federal de Educação, Ciência e Tecnologia do Pará, Campus Abaetetuba, R. Rio Grande do Sul 3322, 68440-000, Abaetetuba, PA, Brasil jeferson.m.costa@hotmail.com

${ }^{2}$ Universidade Federal do Pará, Campus Bragança, Lab. Biologia Vegetal, Al. Leandro Ribeiro s/n, 68370-000, Bragança, PA, Brasil. pietrobomsilva@yahoo.com 
desde o nível do mar até quase o limite da vegetação altimotana. Apresentam uma grande variedade de formas de vida que incluem plantas terrestres, epífitas, rupícolas, aquáticas, hemiepífitas e trepadeiras, variando desde minúsculas herbáceas até formas arborescentes com $20 \mathrm{~m}$ de altura (Windisch 1992). Entretanto, apesar de ocorrerem em vários ecossistemas, com diferentes formas de vida, o que pressupõe uma ampla variedade de adaptações, a maior parte das espécies (cerca de 3/4) é encontrada apenas nos trópicos e, destas, cerca de 1/3 cresce como epífitas (Zuquim et al. 2008).

O conhecimento sobre a flora de plantas vasculares sem sementes do estado do Pará obteve grandes avanços nos últimos sete anos. Várias espécies nunca antes referidas para o estado foram coletadas e citadas em trabalhos recentemente publicados, como os realizados na Área de Pesquisas Ecológicas do Guamá (Rodrigues et al. 2004), no Parque Ambiental de Belém (Costa et al. 2006) e na Ilha de Mosqueiro (Costa \& Pietrobom 2007), áreas que incluem florestas de terra firme (o tipo predominante), várzea e igapó. Alguns inventários, no entanto, restringiram-se a apenas um tipo de ecossistema, como no Bosque Rodrigues Alves - Jardim Botânico da Amazônia, constituído por floresta de terra firme (Maciel et al. 2007), na Ilha do Combu, representada unicamente por floresta de várzea (Ferreira et al. 2009) e nas restingas das Ilhas de Algodoal e Maiandeua (Costa 2009). Com exceção deste último, realizado no município de Maracanã, os demais estudos foram desenvolvidos em áreas florestais de Belém, que estão sob grande pressão antrópica (Leão et al. 2008). Mesmo assim, estes fragmentos florestais apresentaram mais de 15 novas ocorrências para o estado, incluindo novos registros para a Região Norte e para o Brasil, o que mostra a importância das florestas urbanas para a conservação da biodiversidade local e regional.

A região metropolitana de Belém abrange além da capital paraense os municípios de Ananindeua, Marituba, Benevides e Santa Bárbara do Pará. Embora componham uma das áreas mais afetadas pela presença humana na Amazônia, estes ainda apresentam remanescentes florestais preservados, com poucos registros de samambaias e licófitas. Uma dessas áreas compreende o Parque Ecológico do Gunma (PEG), localizado no município de Santa Bárbara do Pará, e cuja flora fanerogâmica é bem conhecida, sendo constituída por espécies de alto valor conservacionista, paisagístico, biológico e econômico. Neste Parque, foram registradas espécies arbóreas consideradas raras, como Vouacapoua americana Aubl. (acapu),
Manilkara huberi (Ducke) Chevalier (maçaranduba), Tabebuia impetiginosa (Mart. ex DC.) Standl. (ipêroxo), entre outras (Almeida et al. 2003; Videira 2007). No entanto, os inventários botânicos pretéritos realizados no PEG não abrangeram a sua flora de plantas vasculares sem sementes.

Desta forma, o objetivo do presente estudo foi inventariar as espécies de samambaias e licófitas do Parque Ecológico do Gunma (Santa Bárbara do Pará, estado do Pará, Brasil), além de caracterizar hábito, habitat, ambiente de ocorrência e padrão de distribuição geográfica das espécies.

\section{Material e Métodos}

O Parque Ecológico do Gunma (PEG) está localizado no município de Santa Bárbara do Pará, região metropolitana de Belém (RM-Belém), nordeste do estado do Pará (Fig. 1a-c). Possui cerca de 540 ha $\left(01^{\circ} 13^{\prime} 00,86^{\prime \prime} S-48^{\circ} 17^{\prime} 41,18^{\prime \prime} \mathrm{W}\right)$, com acesso no km 18 da Rodovia PA-391, sentido Belém-Ilha de Mosqueiro, sendo gerenciado pela Associação Gunma Kenjin-Kai do norte do Brasil (Almeida et al. 2003).

No PEG a vegetação predominante é floresta ombrófila densa de terra firme, além de florestas secundárias (capoeiras), igapós e várzeas (Almeida et al. 2003). O parque faz parte da Planície Amazônica, com pouca declividade mesmo próximo aos cursos d'água, e apresentando os solos latossolos amarelos álicos, concrecionário laterítico álico e gley pouco húmido (RADAMBRASIL 1974).

Quanto à hidrologia, o PEG apresenta ao sul o igarapé Tracuateua e ao nordeste o igarapé Tauriê (Fig. 1c), os quais formam os ambientes de várzea e de igapó ocorrentes na área (Almeida et al. 2003). A região apresenta clima do tipo $\mathrm{Af}_{\mathrm{i}}$ - tropical úmido (classificação de Köeppen), temperatura média anual em torno de $26^{\circ} \mathrm{C}$, com mínima de $22^{\circ} \mathrm{C}$ e máxima de $31^{\circ} \mathrm{C}$, e precipitação pluviométrica anual variando de 2500 a $3000 \mathrm{~mm}$, com umidade relativa do ar atingindo cerca de 85\% (SUDAM 1984).

As coletas foram realizadas nos meses de abril, maio e agosto de 2005, maio de 2006 e em julho de 2008, sendo uma por mês, totalizando cinco expedições ao parque. Durante a etapa de campo, foram percorridos os diversos ambientes contidos nas formações vegetacionais do PEG, objetivando a coleta das espécies e o registro de seus hábitos, habitats e ambientes de ocorrência. As amostras foram coletadas e herborizadas seguindo a metodologia especificada por Windisch (1992). 

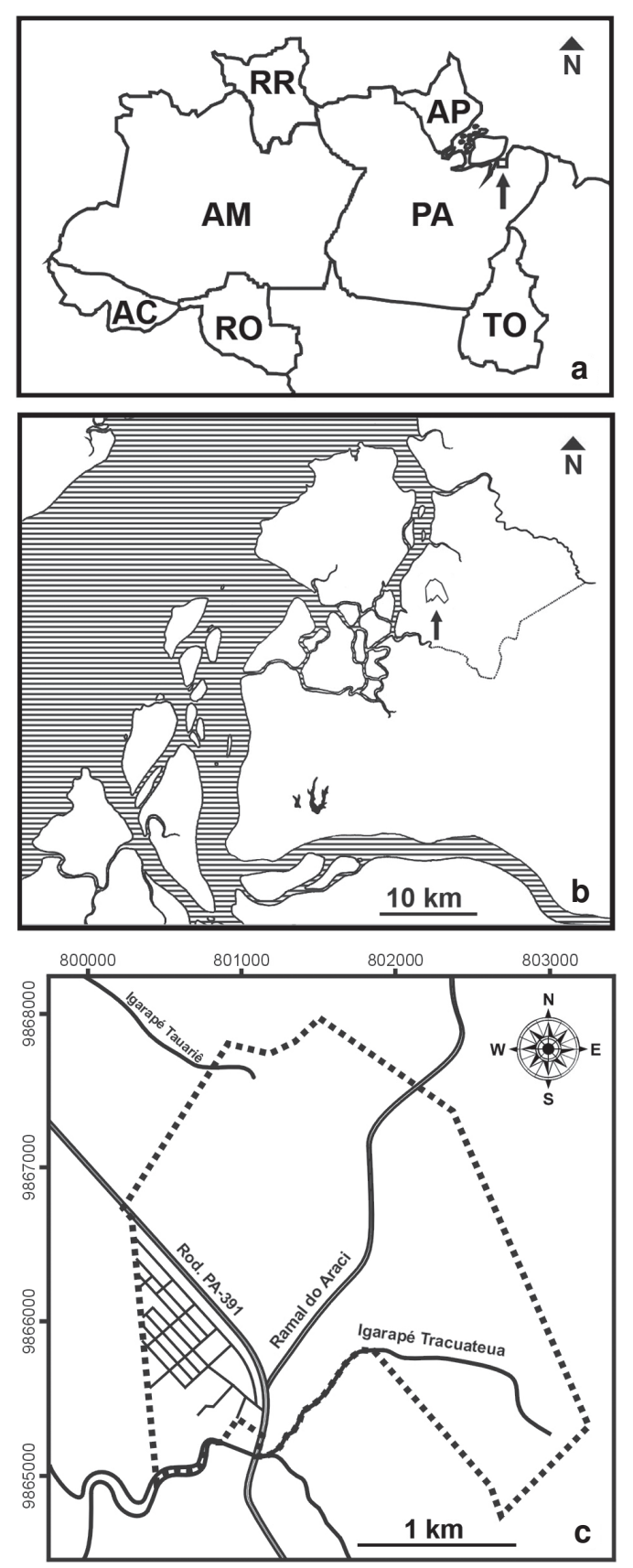

Figura 1 - a. localização da região metropolitana de Belém (seta) em relação ao estado do Pará, Região Norte do Brasil; b. localização do Parque Ecológico do Gunma (seta) em relação ao Município de Santa Bárbara do Pará, região metropolitana de Belém; c. delimitação do Parque Ecológico do Gunma (---).

Figure 1 - a. localization of the metropolitan area of Belém (arrow) in state of Pará, northern Brazil; b. localization of the Parque Ecológico do Gunma (arrow) in municipality of Santa Bárbara do Pará, metropolitan area of Belém; c. limits of the Parque Ecológico do Gunma ("-").
A identificação dos exemplares coletados foi realizada com base em chaves de identificação e textos especializados, como Alston et al. (1981), Barrington (1978), Boer (1962), Kramer (1957), Labiak \& Prado (2003, 2005), Mickel \& Smith (2004), Moran (2000), Moran \& Riba (1995), Prado (2005), Prado \& Labiak (2005), Prado \& Moran (2008), Smith (1986, 1995), Sylvestre (2001), Tryon \& Stolze (1989a-b, 1991, 1994) e Windisch (1978, 1996b). Os nomes dos autores das espécies foram padronizados de acordo com PichiSermolli (1996). O material testemunho foi incorporado ao acervo do herbário MG e, sempre que possível, com duplicatas enviadas para os herbários MBM, PACA, RB, SP e UB (Holmgren et al. 1990).

As licófitas foram circunscritas de acordo com Kramer \& Green (1990) e, para as monilófitas (referidas aqui como samambaias), seguiu-se a circunscrição de famílias proposta por Smith et al. (2006), exceto para Cyatheaceae onde seguiu-se Lellinger (1987), e Hymenophyllaceae, para a qual foi adotada a classificação proposta por Ebihara et al. (2006). Os táxons compreendidos em cada um desses grupos são citados a partir de família e organizados em ordem alfabética.

As espécies foram classificadas quanto ao hábito em herbáceas, arborescentes e trepadeiras. Em relação aos habitats, foram consideradas como terrestres, epífitas ou hemiepífitas. Os ambientes de ocorrência reconhecidos na área foram subbosque, margem da mata e clareira.

A análise da distribuição geográfica das espécies segue a classificação proposta por Parris (2001) e Moran \& Smith (2001), com modificações de Schwartsburd \& Labiak (2007), segundo os quais as espécies podem ser classificadas em: circum-antárticas (espécies presentes na América, África, Ásia e/ou Oceania), atlânticas (América, África e eventualmente ilhas do Oceano Atlântico), pacíficas (América, Ásia e/ou Oceania), americanas (Américas do Sul, Central e eventualmente do Norte) e sul-americanas (apenas nos países da América do Sul). A distribuição geográfica das espécies nos países do continente americano, bem como sua ocorrência em outros continentes, foi feita com base em Moran \& Riba (1995), Berry et al. (1995), Mickel \& Smith (2004) e nas demais obras utilizadas na identificação das espécies.

\section{Resultados e Discussão}

No Parque Ecológico do Gunma (PEG) foram registradas 56 espécies, sendo três espécies de licófitas, distribuídas em dois gêneros e duas famílias, e 53 espécies de samambaias, pertencentes a 26 
Tabela 1 - Lista das espécies de samambaias e licófitas do Parque Ecológico do Gunma (PEG), município de Santa Bárbara, estado do Pará, Brasil. Ambientes de ocorrência: MM - Margem da mata; SB - Sub-bosque; CL - Clareira. $\left({ }^{+}\right)$Espécies com primeira indicação em Unidade de Conservação na região metropolitana de Belém. Material selecionado depositado em MG.

Table 1 - List of ferns and lycophytes species of the Parque Ecológico do Gunma (PEG), municipality of Santa Bárbara do Pará, state of Pará, Brazil. Environments types: MM - Forest border; SB - Understory; CL - Clearing. (+ ) Species reported for the first time in a conservation unit area within the metropolitan area of Belém. Voucher deposited at MG.

\begin{tabular}{|c|c|c|c|c|}
\hline Táxon & Habitat & Ambiente & $\begin{array}{c}\text { Distribuição } \\
\text { geográfica }\end{array}$ & $\begin{array}{c}\text { Material } \\
\text { selecionado }\end{array}$ \\
\hline
\end{tabular}

\section{LICÓFITAS}

LYCOPODIACEAE

Lycopodiella cernua (L.) Pic. Serm.

Terrestre

MM

Circum-Antártica

Pietrobom 5984

SELAGINELLACEAE

Selaginella conduplicata Spring

Selaginella producta Baker ${ }^{+}$

Terrestre

Terrestre

$\mathrm{SB} / \mathrm{MM}$

$\mathrm{SB}$

\section{SAMAMBAIAS}

\section{ASPLENIACEAE}

Asplenium juglandifolium Lam. ${ }^{+}$

Asplenium serratum L.

\section{BLECHNACEAE}

Blechnum serrulatum Rich.

Salpichlaena hookeriana (Kuntze) Alston ${ }^{+}$

OO CYATHEACEAE

Cyathea cyatheoides (Desv.) K.U. Kramer

Cyathea microdonta (Desv.) Domin

Cyathea surinamensis (Miq.) Domin

\section{DRYOPTERIDACEAE}

Cyclodium heterodon (Schrad.) T. Moore var. abbreviatum (C. Presl) A.R. Sm. Terrestre

Cyclodium meniscioides (Willd.) C. Presl var. meniscioides

Epífita

Epífita

SB

SB

Terrestre

MM

Terrestre

SB

Terrestre

Terrestre

Terrestre

$\mathrm{SB} / \mathrm{CL}$

SB/MM

$\mathrm{SB}$

SB

Terrestre

Epífita

\section{Sul-Americana}

Americana

Americana

Americana

Pacífica

Sul-Americana

Sul-Americana

Americana

Americana

Sul-Americana

Sul-Americana

Sul-Americana 


\begin{tabular}{|c|c|c|c|c|}
\hline Táxon & Habitat & Ambiente & $\begin{array}{l}\text { Distribuição } \\
\text { geográfica }\end{array}$ & $\begin{array}{l}\text { Material } \\
\text { selecionado }\end{array}$ \\
\hline Elaphoglossum flaccidum (Fée) T. Moore ${ }^{+}$ & Epífita & SB & Americana & Pietrobom et al. 7727 \\
\hline Elaphoglossum laminarioides (Bory ex Fée) T. Moore ${ }^{+}$ & Epífita & $\mathrm{SB} / \mathrm{CL}$ & Sul-Americana & Pietrobom 5979 \\
\hline Elaphoglossum luridum (Fée) Christ & Epífita & $\mathrm{SB} / \mathrm{CL}$ & Americana & Pietrobom 5977 \\
\hline Elaphoglossum obovatum Mickel $^{+}$ & Epífita & $\mathrm{SB} / \mathrm{CL}$ & Sul-Americana & Pietrobom et al. 7733 \\
\hline Elaphoglossum styriacum Mickel $^{+}$ & Epífita & SB & Sul-Americana & Pietrobom 8258 \\
\hline \multicolumn{5}{|l|}{ HYMENOPHYLLACEAE } \\
\hline Hymenophyllum abruptum Hook. & Epífita & SB & Americana & Pietrobom et al. 5896 \\
\hline Hymenophyllum hirsutum (L.) Sw. ${ }^{+}$ & Epífita & $\mathrm{SB} / \mathrm{CL}$ & Americana & Pietrobom \& Sanjuan 6094 \\
\hline Hymenophyllum polyanthos (Sw.) Sw. & Epífita & $\mathrm{SB} / \mathrm{CL}$ & Circum-Antártica & Pietrobom \& Sanjuan 6097 \\
\hline Didymoglossum angustifrons Fée & Epífita & SB & Americana & Pietrobom 5950 \\
\hline Didymoglossum kapplerianum (J.W. Sturm) Ebihara \& Dubuisson & Epífita & SB & Americana & Pietrobom 5988 \\
\hline Didymoglossum pinnatinervium (Jenman) Pic. Serm. & Epífita & SB & Americana & Pietrobom 5989 \\
\hline Didymoglossum punctatum (Poir.) Desv. & Epífita & $\mathrm{SB} / \mathrm{CL}$ & Americana & Pietrobom 5960 \\
\hline Polyphlebium diaphanum (Kunth) Ebihara \& Dubuisson & Epífita & SB & Americana & Pietrobom et al. 6268 \\
\hline Trichomanes accedens $\mathrm{C}$. Presl ${ }^{+}$ & Epífita & SB & Americana & Pietrobom et al. 6263 \\
\hline Trichomanes ankersii C. Parker ex Hook. \& Grev. & Hemiepífita & SB & Americana & Pietrobom \& Sanjuan 6076 \\
\hline Trichomanes arbuscula Desv. & Epífita & SB & Americana & Pietrobom \& Sanjuan 6090 \\
\hline Trichomanes hostmannianum (Klotzsch) Kunze ${ }^{+}$ & Terrestre & SB & Sul-Americana & Pietrobom \& Sanjuan 6061 \\
\hline Trichomanes pedicellatum Desv. & Hemiepífita & SB & Americana & Pietrobom et al. 7719 \\
\hline Trichomanes pinnatum Hedw. & Terrestre & SB & Americana & Pietrobom et al. 7725 \\
\hline Trichomanes trollii Bergdolt ${ }^{+}$ & Terrestre & SB & Sul-Americana & Pietrobom et al. 6259 \\
\hline \multicolumn{5}{|l|}{ LINDSAEACEAE } \\
\hline Lindsaea lancea var. falcata (Dryand.) Rosenst. & Terrestre & SB & Americana & Pietrobom et al. 7717 \\
\hline Lindsaea lancea (L.) Bedd. var . lancea & Terrestre & SB & Americana & Pietrobom et al. 7712 \\
\hline \multicolumn{5}{|l|}{ LOMARIOPSIDACEAE } \\
\hline Lomariopsis prieuriana Fée & Hemiepífita & $\mathrm{SB} / \mathrm{CL}$ & Americana & Pietrobom et al. 6255 \\
\hline Nephrolepis hirsutula (G. Forst.) C. Presl & Epífita & MM & Pacífica & Pietrobom 5954 \\
\hline
\end{tabular}




\begin{tabular}{|c|c|c|c|c|}
\hline Táxon & Habitat & Ambiente & $\begin{array}{l}\text { Distribuição } \\
\text { geográfica }\end{array}$ & $\begin{array}{l}\text { Material } \\
\text { selecionado }\end{array}$ \\
\hline \multicolumn{5}{|l|}{ MARATTIACEAE } \\
\hline Danaea simplicifolia Rudge & Terrestre & SB & Americana & Pietrobom 5971 \\
\hline \multicolumn{5}{|l|}{ METAXYACEAE } \\
\hline Metaxya rostrata (Kunth) C. Presl & Terrestre & $\mathrm{SB} / \mathrm{CL}$ & Americana & Pietrobom 5994 \\
\hline \multicolumn{5}{|l|}{ OLEANDRACEAE } \\
\hline Oleandra articulata (Sw.) C. Presl ${ }^{+}$ & Epífita & $\mathrm{CL}$ & Americana & Pietrobom 5975 \\
\hline \multicolumn{5}{|l|}{ POLYPODIACEAE } \\
\hline Campyloneurum phyllitidis (L.) C. Presl & Epífita & $\mathrm{SB} / \mathrm{CL}$ & Americana & Pietrobom 5966 \\
\hline Cochlidium serrulatum (Sw.) L.E. Bishop & Epífita & $\mathrm{SB} / \mathrm{CL}$ & Atlântica & Pietrobom \& Sanjuan 6093 \\
\hline Lellingeria suspensa (L.) A.R. Sm. \& R.C. Moran & Epífita & MM & Americana & Pietrobom \& Sanjuan 6095 \\
\hline Microgramma lycopodioides (L.) Copel. & Epífita & SB/MM & Americana & Pietrobom et al. 7734 \\
\hline Microgramma reptans (Cav.) A.R. Sm. & Epífita & SB/MM & Americana & Pietrobom \& Sanjuan 6077 \\
\hline Microgramma thurnii (Baker) R.M. Tryon & Epífita & SB & Sul-Americana & Pietrobom et al. 6252 \\
\hline Serpocaulon triseriale (Sw.) A.R. Sm. & Epífita & MM & Americana & Pietrobom 8259 \\
\hline \multicolumn{5}{|l|}{ PTERIDACEAE } \\
\hline Adiantum glaucescens Klotzsch & Terrestre & SB & Sul-Americana & Pietrobom 5969 \\
\hline Adiantum paraense Hieron. ${ }^{+}$ & Terrestre & SB & Sul-Americana & Pietrobom et al. 6257 \\
\hline Adiantum tomentosum Klotzsch ${ }^{+}$ & Terrestre & SB & Sul-Americana & Pietrobom \& Sanjuan 6084 \\
\hline Anetium citrifolium (L.) Splitg. & Epífita & SB & Americana & Pietrobom \& Sanjuan 6096 \\
\hline Hecistopteris pumila (Spreng.) J. Sm. & Epífita & SB & Americana & Pietrobom \& Sanjuan 6092 \\
\hline Polytaenium guayanense (Hieron.) Alston & Epífita & SB & Americana & Pietrobom et al. 6251 \\
\hline \multicolumn{5}{|l|}{ TECTARIACEAE } \\
\hline Triplophyllum angustifolium Holttum ${ }^{+}$ & Terrestre & SB & Sul-Americana & Pietrobom \& Sanjuan 6082 \\
\hline Triplophyllum dicksonioides (Fée) Holttum ${ }^{+}$ & Terrestre & SB & Sul-Americana & Pietrobom et al. 6253 \\
\hline Triplophyllum funestum (Kunze) Holttum & Terrestre & SB & Americana & Pietrobom 5981 \\
\hline Triplophyllum hirsutum (Holttum) J. Prado \& R.C. Moran ${ }^{+}$ & Terrestre & SB & Americana & Pietrobom \& Sanjuan 6072 \\
\hline
\end{tabular}


Tabela 2 - Lista de inventários de samambaias e licófitas realizados no estado do Pará. $\mathrm{N}^{\circ}$ Esp. - número de espécies; habitat: T - Terrestre, E - Epífita, H - Hemiepífita, R - Rupícola, A - Aquática flutuante.

Table 2 - List of ferns and lycophytes inventories conducted in state of Pará. $N^{o}$ Esp. - number of species; habitat: $\mathrm{T}$ - Terrestrial, E - Epiphytic, H - Hemiepiphytic, R - Lithophyte, A - Floating aquatic.

\begin{tabular}{|c|c|c|c|c|c|c|c|c|c|}
\hline \multirow[t]{2}{*}{ Área inventariada } & \multirow[t]{2}{*}{ Município } & \multirow[t]{2}{*}{ Tamanho } & \multirow[t]{2}{*}{$\mathbf{N}^{0}$ Esp. } & \multicolumn{5}{|c|}{ Habitat } & \multirow[t]{2}{*}{ Referência } \\
\hline & & & & $\mathbf{T}$ & $\mathbf{E}$ & $\mathbf{H}$ & $\mathbf{R}$ & $\mathbf{A}$ & \\
\hline Parque Ecológico do Gunma & $\begin{array}{l}\text { Santa Bárbara } \\
\text { do Pará }\end{array}$ & 540 ha & 56 & 23 & 30 & 3 & - & - & Neste estudo \\
\hline Ilha de Mosqueiro & Belém & 22.000 ha & 70 & 39 & 33 & 4 & - & - & Costa \& Pietrobom 2007 \\
\hline $\begin{array}{l}\text { Restingas da APA } \\
\text { Algodoal-Maiandeua }\end{array}$ & Maracanã & 2.378 ha & 10 & 5 & 2 & - & - & 3 & Costa 2009 \\
\hline Ilha do Combu & Belém & $1.500 \mathrm{ha}$ & 26 & 10 & 15 & 2 & - & - & Ferreira et al. 2009 \\
\hline Parque Ambiental de Belém & Belém & 1.340 ha & 49 & 27 & 20 & 4 & 2 & 1 & Costa et al. 2006 \\
\hline Bosque Rodrigues Alves & Belém & 15 ha & 39 & 17 & 21 & - & 4 & 1 & Maciel et al. 2007 \\
\hline $\begin{array}{l}\text { Área de Pesquisas Ecológicas } \\
\text { do Guamá }\end{array}$ & Belém & 12 ha & 39 & 20 & 16 & 3 & - & - & Rodrigues et al. 2004 \\
\hline
\end{tabular}

gêneros e 13 famílias (Tab. 1). Trata-se da segunda área com maior riqueza de espécies na região metropolitana de Belém, sendo neste aspecto inferior apenas à Ilha de Mosqueiro (Tab. 2), área distrital do município de Belém, onde foram registradas 70 espécies (Costa \& Pietrobom 2007). Ainda assim, a riqueza de espécies do Parque pode ser considerada significativa, uma vez que Mosqueiro possui uma maior variabilidade de ambientes e área 40 vezes maior que a do PEG (Tab. 2).

O número de espécies registradas no parque foi maior do que em outras quatro áreas inventariadas em Belém (Tab. 2): a Área de Pesquisas Ecológicas do Guamá (Rodrigues et al. 2004) e o Bosque Rodrigues Alves - Jardim Botânico da Amazônia (Maciel et al. 2007), nas quais a menor quantidade de espécies se deve provavelmente ao tamanho reduzido de suas respectivas áreas, que são pelo menos 35 vezes menores que a do PEG; o Parque Ambiental de Belém (Costa et al. 2006), onde a menor riqueza de espécies deve estar associada ao seu maior grau de alteração antrópica, visto que o PEG, por estar mais distante do centro urbano, vem sofrendo menos com a pressão humana e possui uma vegetação mais conservada; e a Ilha do Combu (Ferreira et al. 2009), cuja pequena representatividade de espécies se atribui ao ambiente de várzea, que constitui a totalidade de seu território, enquanto no PEG ocorre uma maior variedade de tipos vegetacionais. A riqueza específica do parque foi maior também que a registrada nas restingas da APA Algodoal-Maiandeua, município de Maracanã/PA (Costa 2009) (tab. 2). Esta constatação pode estar relacionada ao fato de, em geral, samambaias e licófitas serem pouco tolerante à potenciais condições de déficit hídrico (Holttum 1967), assim, ambientes florestais, como é o caso do PEG, apresentam uma maior riqueza de espécies do que as formações não-florestais, como as restingas.

São citados dois novos registros, Lellingeria suspensa para o estado do Pará e Polyphlebium diaphanum para a Região Norte (Amazônia brasileira). Era esperado que estas espécies ocorressem no Pará, pois Lellingeria suspensa está amplamente distribuída nos países ao Norte da América do Sul e no Brasil, ocorre no Amapá, Bahia, Espírito Santo e São Paulo (Labiak \& Prado 2005). Polyphlebium diaphanum também ocorre nos países amazônicos (Smith 1995; Pacheco 1995) e no Brasil era citada para as Regiões Sudeste e Sul, em Minas Gerais (Figueiredo \& Salino 2005), São Paulo (Salino 1996) e Paraná (Schwartsburd \& Labiak 2007).

A flora fanerogâmica do PEG apresentou espécies consideradas raras por não apresentarem mais populações naturais em outras áreas da RMBelém (Almeida et al. 2003). O mesmo foi observado em relação a algumas espécies de samambaias, como Hymenophyllum abruptum que possui somente um registro de ocorrência no Pará referido há mais de 30 anos por Tryon \& Conant (1975).

Outro aspecto que demonstra a importância do parque para a conservação da flora de samambaias e licófitas da região é o fato de que, do total de espécies registradas, 17 espécies ainda não tinham sido coletadas em áreas protegidas da RM-Belém (Tab. 1). 
Entre estas espécies estão Elaphoglossum obovatum, E. styriacum, Trichomanes trollii, Salpichlaena hookeriana e Triplophyllum angustifolium, citadas há pouco tempo como novos registros para o estado do Pará (Costa \& Pietrobom 2007).

As famílias mais representativas foram Hymenophyllaceae (15 spp.), Dryopteridaceae (8 spp.), Polypodiaceae (7 spp.) e Pteridaceae (6 spp.), em conformidade com os dados obtidos nas demais áreas inventariadas de Belém (Rodrigues et al. 2004; Costa et al. 2006; Maciel et al. 2007; Costa \& Pietrobom 2007). Em trabalhos florísticos realizados no estado do Amazonas estas famílias também são as mais ricas, como na Reserva Ducke (Hopkins 2005) e na Reserva Biológica de Uatumã (Zuquim et al. 2008), ambas em Manaus, e nas florestas inundáveis do alto Rio Negro, em Santa Isabel do Rio Negro (Freitas \& Prado 2005).

No PEG, Hymenophyllaceae apresentou a maior riqueza específica já registrada em relação aos outros inventários locais feitos na Amazônia brasileira (Edwards 1998; Rodrigues et al. 2004; Freitas \& Prado 2005; Hopkins 2005; Costa et al. 2006; Maciel et al. 2007; Costa \& Pietrobom 2007; Zuquim et al. 2008; Ferreira et al. 2009; Costa 2009), sendo que das 18 espécies desta família registradas em Belém somente três não foram coletadas no Parque, Vandenboschia repens Schott ex J.W. Sturm, Trichomanes vittaria DC. ex Poir. (Rodrigues et al. 2004) e Didymoglossum krausii (Hook. \& Grev.) C. Presl (Maciel et al. 2007; Ferreira et al. 2009). O gênero mais representativo foi Trichomanes L. com sete espécies (Tab. 1). A elevada riqueza específica de Dryopteridaceae está relacionada à elevada quantidade de espécies de Elaphoglossum Schott. ex J. Sm. (6 spp.), o segundo gênero mais representativo (Tab. 1), sendo composto pelas mesmas espécies referidas para a Ilha de Mosqueiro (Costa \& Pietrobom 2007).

$\mathrm{Na}$ área inventariada, foram coletadas três espécies arborescentes, Cyathea cyatheoides, $C$. microdonta e C. surinamensis. Assim, das quatro espécies registradas com esse hábito nos remanescentes florestais de Belém, somente $C$. pungens (Willd.) Domin (Costa \& Pietrobom 2007) não ocorre no PEG. Apenas uma espécie trepadeira foi coletada no Parque, Salpichlaena hookeriana, a qual, segundo Costa \& Pietrobom (2007), apresenta pouca tolerância a alterações ambientais.

Quanto ao habitat das espécies coletadas no PEG, 30 são epífitas, 23 são terrestres e três são hemiepífitas (Tab. 1). O predomínio de espécies epífitas em relação às terrestres (Tab. 2) foi constatado também em florestas de igapó (Rodrigues et al. 2004; Freitas \&
Prado 2005), várzea (Ferreira et al. 2009) e em algumas áreas de terra firme (Maciel et al. 2007). Emáreas mais extensas nas quais a floresta de terra firme é o tipo predominante de vegetação, enquanto igapós e várzeas ocupam áreas menores, foram as espécies terrestres que estiveram em maior número (Costa $e t$ al. 2006; Costa \& Pietrobom 2007), tabela 2. A elevada riqueza de espécies epífitas registrada no Parque está relacionada às famílias mais representativas, uma vez que Polypodiaceae, representada exclusivamente por samambaias epífitas, e Hymenophyllaceae, Dryopteridaceae e Pteridaceae, com pelo menos metade de suas espécies crescendo em habitat epifítico, apresentaram mais de $80 \%$ das espécies epífitas registradas no PEG. Contudo, a representatividade de samambaias nesse tipo de habitat deve ainda estar subestimada, pois até o momento não foi usada uma metodologia direcionada à amostragem de epífitas ocorrentes em dossel.

As espécies ocorrentes na área encontravamse associadas à floresta e distribuídas ao longo de três ambientes reconhecidos como clareiras, margem das matas e sub-bosque. Foi no sub-bosque que se registrou o maior número de espécies (50 spp.), das quais 35 ocorreram exclusivamente nesse ambiente, enquanto nas clareiras e margens das matas não ocorreram mais que cinco espécies exclusivas (Tab. 1). A preferência destas plantas pelo interior das matas (sub-bosques) em detrimento a outros ambientes associados ou não às formações florestais foi constatada por todos os demais estudos feitos na região metropolitana de Belém (Rodrigues et al. 2004; Costa et al. 2006; Maciel et al. 2007; Costa \& Pietrobom 2007).

A maioria das espécies registradas no parque apresenta distribuição americana (34 spp.), representando o dobro de espécies sul-americanas (17 spp.). Entretanto, entre as espécies consideradas americanas, algumas têm uma distribuição restrita à América do Sul e Trinidad: Cyathea surinamensis, Elaphoglossum flaccidum (que ocorre também em Tobago), Didymoglossum pinnatinervium, Trichomanes accedens, T. arbuscula, T. pedicellatum e Danaea simplicifolia. Poucas espécies ocorrem em outros continentes, sendo duas circum-antárticas (Lycopodiella cernua e Hymenophyllum polyanthos), duas pacíficas (Blechnum serrulatum e Nephrolepis hirsutula) e uma atlântica (Cochlidium serrulatum).

Com os resultados obtidos, pode-se afirmar que o Parque Ecológico do Gunma, mesmo localizado numa área de grande pressão antrópica, constitui uma área floristicamente importante, não só em relação à já conhecida flora fanerogâmica, mas agora 
também no que tange à flora de plantas vasculares sem sementes da região metropolitana de Belém e do estado do Pará. Essa importância é evidenciada pela grande riqueza de espécies, entre as quais algumas são raras, como Hymenophyllum abruptum, registrada pela última vez no Pará e talvez no Brasil, há mais de 30 anos. Pode ser mencionada também a ocorrência de dois novos registros, um para o estado (Lellingeria suspensa) e um para a Região Norte Amazônia brasileira (Polyphlebium diaphanum), bem como a ocorrência de 23 espécies ainda não coletadas em áreas protegidas da Grande Belém.

\section{Agradecimentos}

Ao Museu Paraense Emílio Goeldi (MPEG) por fornecer suas instalações e infra-estrutura; à Associação Gunma Kenjin-Kai do Norte do Brasil por autorizar e apoiar as coletas na área do Parque; à Bióloga Priscila Sanjuan de Medeiros o auxílio no trabalho de campo, motivo pelo qual também se deve agradecer aos alunos que cursaram a disciplina Taxonomia de Pteridófitas ofertada pelo curso de Mestrado em Botânica do MPEG; ao Biólogo Luiz Armando de Araújo Góes Neto a elaboração do Abstract; e aos revisores e editores as importantes sugestões para a melhoria deste artigo.

\section{Referências}

Almeida, S.S.; Amaral, D.D. \& Silva, A.S.L. 2003. Projeto: Inventário florístico e análise fitossociológica dos ambientes do Parque Ecológico do Gunma, município de Santa Bárbara, PA. Relatório Técnico Final. Belém: MPEG-CBO/CTBrasil-MCT/JICA. 177p.

Alston, A.H.G.; Jermy, A.C. \& Rankin, J.M. 1981. The genus Selaginella in tropical South America. Bulletin of the British Museum (Natural History). Botany 9: 233-330.

Barrington, D.S. 1978. A revision of the genus Trichipteris. Contributions of the Gray Herbarium of Harvard University 208: 3-93.

Berry, P.E.; Holst, B.K. \& Yatskievych, K. 1995. Pteridophytes, Spermatophytes: Acanthaceae-Araceae. In: Steyermark, J.S.; Berry, P.E. \& Holst, B.K. (eds.). Flora of the Venezuelan Guayana. Vol. 2. Missouri Botanical Garden, Timber Press. Pp. 1-706.

Boer, J.G.W. 1962. The new world species of Thichomanes sect. Didymoglossum and Microgonium. Acta Botanica Neerlandica 11:277-330.

Costa, J.M. 2009. As Pteridófitas. In: Jardim, M.A.G. (org.). Diversidade biológica das áreas de proteção ambiental Ilhas do Combu e Algodoal-Maiandeua, Pará, Brasil. MPEG/MCT/CNPq, Belém. Pp. 213-226.

Costa, J.M. \& Pietrobom, M.R. 2007. Pteridófitas (Lycophyta e Monilophyta) da Ilha de Mosqueiro, município de Belém, estado do Pará, Brasil. Boletim do Museu Paraense Emílio Goeldi. Ciências Naturais 2: 45-56.

Costa, J.M.; Souza, M.G.C. \& Pietrobom, M.R. 2006. Levantamento florístico das pteridófitas (Lycophyta e Monilophyta) do Parque Ambiental de Belém (Belém, Pará, Brasil). Revista de Biologia Neotropical 3: 4-12.

Ebihara, A.; Dubuisson, J-Y.; Iwatsuki, K.; Hennequin, S. \& Ito, M. 2006. A taxonomic revision of Hymenophyllaceae. Blumea 51: 221-280.

Edwards, P.J. 1998. The Pteridophytes of the Ilha de Maracá. In: Milliken, W. \& Ratter, J.A. (eds.). Maracá: the biodiversity and environment of an Amazonian rainforest. John Wiley \& Sons Ltd. Pp. 113-129.

Ferreira, L.S.L.; Costa, J.M. \& Pietrobom, M.R. 2009. As pteridófitas. In: Jardim, M.A.G. (org.). Diversidade biológica das áreas de proteção ambiental Ilhas do Combu e Algodoal-Maiandeua, Pará, Brasil. MPEG/ MCT/CNPq, Belém. Pp. 13-40.

Figueiredo, J.B. \& Salino, A. 2005. Pteridófitas de quatro reservas particulares do patrimônio natural ao sul da região metropolitana de Belo Horizonte, Minas Gerais, Brasil. Lundiana 6: 83-94.

Freitas, C.A.A. \& Prado, J. 2005. Lista anotada das pteridófitas de florestas inundáveis do alto Rio Negro, município de Santa Isabel do Rio Negro, AM, Brasil. Acta Botanica Brasilica 19: 399-403.

Holmgren, P.K.; Holmgren, N.H. \& Banett, L.G. 1990. Index Herbariorum. Part 1: The herbaria of the world. 8ed. Memoirs of the New York Botanical Garden, New York. 452p.

Holttum, R.E. 1967. The ecology of tropical pteridophytes. In: Verdoorn, F. (ed.). Manual of Pteridology. Asher, A. \& Co., Amsterdam. Pp. 420-450.

Hopkins, M.J.G. 2005. Flora da Reserva Ducke, Amazonas, Brasil. Rodriguésia 56: 9-25.

Kramer, K.U. 1957. A revision of the genus Lindsaea in the New World. Acta Botanica Neerlandica 6: 97-290.

Kramer, K.U. \& Green, P.S. (eds.). 1990. Pteridophytes and gymnosperms. Vol. 1. In: Kubitzki, K. (ed.). The families and genera of vascular plants. SpringerVerlag, Berlin. Pp. 1-404.

Labiak, P.H. \& Prado, J. 2003. Grammitidaceae (Pteridophyta) no Brasil comênfase nos gêneros Ceradenia, Cochlidium e Grammitis. Hoehnea 30: 243-283.

Labiak, P.H. \& Prado, J. 2005. As espécies de Lellingeria A.R. Sm. \& R.C. Moran (Grammitidaceae Pteridophyta) do Brasil. Revista Brasileira de Botânica 28: 1-22.

Leão, N.; Alencar, C. \& Veríssimo, A. 2008. Belém Sustentável 2007. Instituto do Homem e Meio Ambiente da Amazônia, Belém. 140p.

Lellinger, D.B. 1987. The disposition of Trichopteris (Cyatheaceae). American Fern Journal 77: 90-94.

Maciel, S; Souza, M.G.C. \& Pietrobom, M.R. 2007. Licófitas e monilófitas do Bosque Rodrigues Alves 
Jardim Botânico da Amazônia, município de Belém, estado do Pará, Brasil. Boletim do Museu Paraense Emílio Goeldi, Ciências Naturais 2: 69-83.

Mickel, J.T. \& Smith, A.R. 2004. The Pteridophytes of Mexico. Memoirs of the New York Botanical Garden 88: 1-1070.

Moran, R.C. 2000. Monograph of the Neotropical species of Lomariopsis (Lomariopsidaceae). Brittonia 52: 55-111.

Moran, R.C. 2008. Diversity, biogeography, and floristics. In: Ranker, T.A. \& Haufler, C.H. (eds.). Biology and evolution of ferns and lycophytes. Cambridge University Press, New York. Pp. 367-394.

Moran, R.C. \& Riba, R. 1995. Psilotaceae a Salviniaceae. In: Davidse, G.; Sousa, M. \& Knapp, S. (ed.). Flora mesoamericana. Universidad Nacional Autónoma de México, Ciudad de México. Vol. 1. Pp. 1-470.

Moran, R.C. \& Smith, A.R. 2001. Phytogeographic relationships between neotropical and AfricanMadagascar pteridophytes. Brittonia 53: 304-351.

Pacheco, L. 1995. Hymenophyllaceae. In: Moran, R.C. \& Riba, R. (ed.). Psilotaceae a Salviniaceae. In: Davidse, G.; Sousa, M. \& Knapp, S. (ed.). Flora mesoamericana. Universidad Nacional Autónoma de México, Ciudad de México. Vol. 1. Pp. 62-83.

Parris, B.S. 2001. Circum-Antarctic continental distribution patterns in pteridophyte species. Brittonia 53: 270-283.

Pichi-Sermolli, R.E.G. 1996. Authors of scientifc names in Pteridophyta. Royal Botanical Garden, Kew. 78p.

Prado, J. 2005. Flora da Reserva Ducke, Amazonas, Brasil: Pteridophyta - Lomariopsidaceae. Rodriguésia 56: 59-66.

Prado, J. \& Labiak, P. H. 2005. Flora da Reserva Ducke, Amazonas, Brasil: Pteridophyta - Vittariaceae. Rodriguésia 56: 108-113.

Prado, J. \& Moran, R.C. 2008. Revision of the neotropical species of Triplophyllum (Tectariaceae). Brittonia 60: 103-130.

Prado, J. \& Moran, R.C. 2009. Checklist of the ferns and lycophytes of Acre State, Brazil. Fern Gazette 18: 230-263.

RADAMBRASIL. 1974. Folha AS. 22 - Belém: geologia, geomorfologia, solos, vegetação e uso potencial da terra. Levantamento de Recursos Naturais. Vol. 5. DNPM, Rio de Janeiro.

Rodrigues, S.T.; Almeida, S.S.; Andrade, L.H.C.; Barros, I.C.L. \& Berg, M.E. van den. 2004. Composição florística e abundância de pteridófitas em três ambientes da bacia do rio Guamá, Belém, Pará, Brasil. Acta Amazonica 34: 35-42.

Salino, A. 1996. Levantamento das pteridófitas da Serra do Cuscuzeiro, Analândia, SP, Brasil. Revista Brasileira de Botânica 19: 173-178.
Schwartsburd, P.B. \& Labiak, P.H. 2007. Pteridófitas do Parque Estadual de Vila Velha, Ponta Grossa, Paraná, Brasil. Hoehnea 34: 159-209.

Smith, A.R. 1986. Revision of the Neotropical fern genus Cyclodium. American Fern Journal 76: 56-98.

Smith, A.R. 1995. Hymenophyllaceae. In: Berry, P.E.; Holst, B.K. \& Yatskievych, K. (eds.). Pteridophytes, spermatophytes: Acanthaceae-Araceae. In: Steyermark, J.S.; Berry, P.E. \& Holst, B.K. (eds.). Flora of the Venezuelan Guayana. Vol. 2. Missouri Botanical Garden, Timber Press. Pp. 159-185.

Smith, A.R.; Pryer, K.M.; Schuettpelz, E.; Korall, P.; Schneider, H. \& Wolf, P.G. 2006. A classification for extant ferns. Taxon 55: 705-731.

SUDAM. 1984. Atlas de climatologia da Amazônia brasileira. Publicação 39. Belém. 125p.

Sylvestre, L.S. 2001. Revisão taxonômica das espécies de Aspleniaceae A.B. Frank ocorrentes no Brasil. Tese de Doutorado. Universidade de São Paulo, São Paulo. 571p.

Tryon, R.M. \& Conant, A.S. 1975. The ferns of Brazilian Amazonica. Acta Amazonica 5: 23-34.

Tryon, R.M. \& Stolze, R.G. 1989a. Pteridophyta of Peru. Part. I. 1. Ophioglossaceae - 12. Cyatheaceae. Fieldiana Botany, new series 20: 1-145.

Tryon, R.M. \& Stolze, R.G. 1989b. Pteridophyta of Peru. Part. II. 13. Pteridaceae - 15. Dennstaedtiaceae. Fieldiana Botany, new series 22: 1-128.

Tryon, R.M. \& Stolze, R.G. 1991. Pteridophyta of Peru. Part. IV. 17. Dryopteridaceae. Fieldiana Botany, new series 27: 1-176.

Tryon, R.M. \& Stolze, R.G. 1994. Pteridophyta of Peru. Part. VI. 22. Marsileaceae - 28. Isoetaceae. Fieldiana Botany, new series 34: 1-123.

Windisch, P.G. 1978. Sphaeropteris (Cyatheaceae). The systematics of the group of Sphaeropteris hirsuta. Memoirs of the New York Botanical Garden 29: 2-22.

Windisch, P.G. 1992. Pteridófitas da região norteocidental do estado de São Paulo: guia para excursões. 2ed. UNESP, São José do Rio Preto. 110p.

Windisch, P.G. 1996a. Towards assaying biodiversity in Brazilian pteridophytes. In: Bicudo, C.E.M. \& Menezes, N.A. Biodiversity in Brazil: a first approach. CNPq, São Paulo. Pp. 109-117.

Windisch, P.G. 1996b. Pteridófitas do estado de Mato Grosso: Hymenophyllaceae. Bradea 6: 400-423.

Videira, M.L. 2007. A biodiversidade do Parque Ecológico do Gunma. In: Silva, L.G.S \& Silva, M.G.S. Caderno de Pesquisa e Educação Ambiental. MCT/MPEG, Belém. Pp. 9-16.

Zuquim, G.; Costa, F.R.C.; Prado, J. \& Tuomisto, H. 2008. Guia de samambaias e licófitas da REBIO Uatumã, Amazônia Central. Design Ed., Manaus. $316 \mathrm{p}$. 